\title{
MOLUSCOS DE IMPORTÂNCIA ECONÔMICA NO BRASIL. III. SUBULINIDAE, LEPTINARIA UNILAMELLATA (ORBIGNY) (MOLLUSCA, GASTROPODA, PULMONATA, STYLOMMATOPHORA) ${ }^{1}$
}

\author{
José Luiz de B. Araújo ${ }^{2}$ \\ Darli Grativol Keller ${ }^{3}$
}

\begin{abstract}
MOLLUSCS OF ECONOMIC IMPORTANCE IN BRASIL. III. SUBULINIDAE, LEPTINARIA UNILAMELLATA (ORBIGNY) (MOLLUSCA, GASTROPODA, PULMONATA, STYLOMMATOPHORA). An anatomic study of Leptinaria unilamellata (Orbigny, 1835) was made emphasizing the genital system, the pallial chamber the radulae and the mandible. The details of these parts characterize better the species and allow more systematic clarity to the group. The importance of the evolutive cycle of the two trematodes, Postharmostomum gallinum (Witenberg, 1923) and Paratanaisia bragai (Santos, 1934) both parasites of domestic birds in Brazil is accentuated.

KEY WORDS. Mollusca, Gastropoda, Pulmonata, Stylommatophora, Leptinaria unilamellata
\end{abstract}

$\mathrm{Na}$ família Subulinidae, de molusco pulmonados terrestres, são incluidas várias espécies capazes de atuarem como hospedeiros intermediários de parasitos de animais domésticos e do homem. Dessas espécies, algumas são assinaladas no Brasil e inclusive já se tendo comprovado suas participações. Contudo, de um modo geral, a participação dessas espécies nestes ciclos, não é bem conhecida, especialmente no Brasil, onde o número de trabalhos, decorrente do escasso número de pesquisadores no assunto, é também reduzido.

Assim, para um perfeito entendimento e segurança das constatações, torna-se necessário, um estudo da anatomia visceral paralelo aos estudos conquiliológicos e biológicos. Estes estudos são poucos, mas existentes e são extremamente necessários para um conhecimento da espécie, mais próximo do que necessitamos, para sua identificação e compreensão do seu envolvimento no ciclo evolutivo de parasitos.

\section{REVISÃO DE LITERATURA}

ORBIGNY (1835) descreve a espécie com se apenas nos caracteres conquiliológicos.

REEVE (1849) descreve as conchas de Achatina octona e Achatina

1) Realizado com auxílio do CNPq.

2) Departamento de Parasitologia Animal, Universidade Federal Rural do Rio de Janeiro, 23851-970 Itaguaí, Rio de Janeiro, Brasil. Bolsista da CAPES.

3) Bióloga. Bolsista do CNPq. 
lamellata, assinalando as particularidades importantes. Da última, atribui a espécie a POTIEZ \& MICHAUD, frizando ser muito semelhante a Achatina funcki Pfeiffer, 1847.

VON MARTENS (1890/1901) considerou na família Stenogyridae os gêneros Subulina, onde inclui entre outras espécies Subulina octona e em Leptinaria unilamellata, não considerou Leptinaria unilamellata. Em todas as espécies apresentou apenas caracteres das conchas.

TRYON \& PILSBRY (1906) estudaram os caracteres morfológicos, especialmente das conchas dos Subulinidae. Caracterizaram os gêneros Subulina e Leptinaria, incluindo as espécies de interesse para o Brasil e a distribuição geográfica por continentes.

BAKER (1913) relacionou, acompanhando uma descrição conquiliológica, várias espécies de moluscos colecionados durante uma expedição efetuada no Brasil.

BAKER (1931) apresentou, como resultado de uma expedição realizada no Brasil, nas regiões Norte, Nordeste e Centro-Oeste, uma lista de espécies de moluscos com descrições conquiliológicas e indicação dos locais onde foram coletados os espécimens.

THIELE (1931) classificou os moluscos a partir dos grandes grupos, caracterizando-os até o nível de subgênero.

PILSBRY (1933) relatou os resultados de uma expedição realizada ao Mato Grosso em 1931, incluindo os achados de Bulimulus tenuissimus e Leptinaria lamellata concentrica.

MORRETES (1943), em sua contribuição ao conhecimento da fauna de moluscos, divulgou os resultados do estudo de uma pequena coleção de moluscos obtida em uma excursão, assinalando vários pontos de coleta. Entre as espécies encontradas, citou Leptinaria lamellata.

HAAS (1952) fez uma distribuição geográfica de espécies de moluscos sul americanos, recebidas do Brasil. Alguns delas tem uma breve descrição conquiliológica.

HAAS (1962), em estudo sobre a fauna de Curaçao e outras ilhas do Caribe, incluiu Subulina octona e Leptinaria lamellata entre os Subulinidae.

DUARTE (1977) descreveu o ciclo de Postharmostomum gallinum no Brasil, constituindo este o trabalho mais completo sobre o parasito; além de Bradybaena similaris e Subulina octona, comuns no Brasil, outras espécies de moluscos entre elas Leptinaria unilamellata, ainda não identificadas como hospedeiras intermediárias de qualquer parasito, nele tiveram comprovada sua participação.

BOFFI (1979), estudando os moluscos brasileiros de interesse médico e econômico, colocou as espécies de interesse veterinário, em parte, como pragas de agricultura, e em parte, como moluscos hospedeiros intermediários de parasitos do homem (que também o são de alguns dos animais domésticos), fazendo um arranjo sistemático dentro de conceitos atualizados. Nesse estudo, são mencionadas espécies que, em nossa conceituação, atuam de diferentes 
maneiras como hospedeiras intermediárias de parasitos de animais domésticos, sendo algumas já comprovadas no Brasil.

OLIVEIRA et al. (1981) catalogaram os moluscos pertencentes à Universidade Federal de Juiz de Fora, incluindo várias espécies de interesse médico-veterinário informando sobre suas procedências

TILLIER (1981) em extenso trabalho acerca dos Subulinideos, faz um arranjo sistemático da família com bases filogenéticas.

KELLER \& ARAUJO (1991) comprovam a participação de L. unilamellata como hospedeira intermediária de Paratanaisia bragai das aves domésticas, no Brasil.

\section{MATERIAL E MÉTODOS}

O estudo foi conduzido na Universidade Federal Rural do Rio de Janeiro (UFRRJ), Itaguaí, Rio de Janeiro, para onde foi transportado o material coletado.

\section{ORIGEM E MANUSEIO DO MATERIAL ESTUDADO}

Para o estudo morfológico dos moluscos, foram utilizados espécimens capturados em vários habitats terrestres no Rio de Janeiro (Valença, Rio das Flores, Três Rios, Paraíba do Sul, Itaguaí).

Os moluscos foram capturados nos locais de suas preferências (sob pedra, em madeira em decomposição, sobre plantas de jardim, em fendas junto a construções, etc). Em maioria, os moluscos ocorriam com maior freqüência após período de chuvas intensas. Os espécimens coletados foram transportados para o laboratório em frascos de vidro com tampa perfurada que permitia uma pequena aeração. No laboratório, eram separados em lotes de acordo com a finalidade. Alguns eram destinados ao estabelecimento de colônias, sendo colocados em caixas onde se procurava reproduzir, tanto quanto possível, seu habitat. Tais moluscos eram utilizados em estudos morfológicos posteriores, observações bionômicas (alimentação, reprodução, etc.) ou infecções experimentais com parasitos para avaliação de sua susceptibilidade. Este material, quando fixado, era depositado na Coleção de Moluscos Hospedeiros Intermediários (Col. Mol. Hosp. Int.), na Área de Parasitologia do Departamento de Biologia Animal, do Instituto de Biologia da Universidade Federal Rural do Rio de Janeiro.

Para os estudos morfológicos, o material, uma vez no laboratório, era dissecado, procurando-se isolar as partes mais importantes para a determinação das espécies, no caso, a cocha, o sistema digestivo (para a retirada da rádula e mandíbula) e o sistema genital.

Os espécimens vivos foram colocados em água e aquecidos a $60^{\circ} \mathrm{C}$ para sacrifício, sendo retiradas as partes moles da concha. Os moluscos foram colocados em solução fisiológica, para serem dissecados. Outras vezes eram dissecados em álcool glicerinado, quando se utilizava este líquido para sua conservação por ocasião da coleta. Quando da dissecação, se um detalhe da 
anatomia topográfica era julgado importante, executavam-se desenhos em câmara clara.

Concluídas as dissecções e separadas as partes de interesse, procedia-se do seguinte modo: o bulbo da rádula era fervido em potassa a $10 \%$ para o isolamento de rádula e mandíbula. As rádulas eram coradas em uma mistura de Vermelho Congo e Orange $\mathbf{G}$ após desidratação na série de alcoois e xilol. Em seguida, eram montadas em bálsamo-do-Canadá entre lâmina e lamínula. $\mathrm{O}$ sistema genital era desidratado na série de álcoois e posteriormente corado em carmin clorídrico, clarificado em creosoto, onde era conservado e estudado.

Abreviaturas usadas:
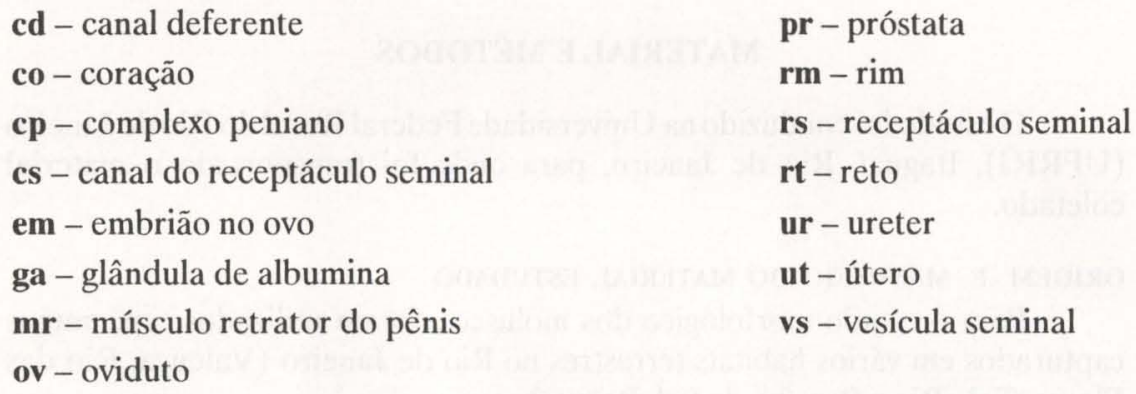

\section{Leptinaria unilamellata (Orbigny, 1835)}

Figs 1-11

Bulimus unilamellata Orbigny, 1835:257.

Tornatellina ferussaci Pfeiffer, 1848:394.

Achatina lamellata Reeve, 1849: sp.97, pl.XVIII.

Tornatellina unilamellata; Pfeiffer, 1859:659-650.

Leptinaria lamellata; Tryon e Pilsbry, 1906:288-289. pl.42, figs 39-40, pl.43, fig. 50.

Leptinaria unilamellata; Wens, 1960:348, fig.1280.

Leptinaria (Leptinaria) lamellata; Haas, 1962:52-53, fig. VII, figs F-G.

Concha (Figs 1,2). É pequena, com 4, $5 \mathrm{~mm}$ e cerca de cinco a 6,5 voltas de espira, com a superfície mostrando as linhas de crescimento não muito marcadas, suturas bem evidentes, mas não muito profundas. Volta corporal com comprimento equivalente a pouco mais que as demais. Abertura ovalada com peristoma cortante, bordo não refletido e desprovido de dentes ou lamelas no bordo externo. Bordo columelar com uma lamela lisa, triangular, baixa e projetada para a abertura. Bordo parietal com uma lamela transversal à abertura e situada a meia distância entre término da lamela columelar e a inserção da última volta.

Camâra palial (Fig. 4). Apresenta poucos vasos, destacando-se apenas a veia pulmonar, que recebe algumas afluentes. Sua origem junto ao pneumóstomo é pouco marcada. O rim é o órgão mais característico para a espécie, nesta região do molusco. Sua forma é alongada e estreita no sentido transversal à câmara paleal. Do lado de sua extremidade columelar, para o ureter primário, 

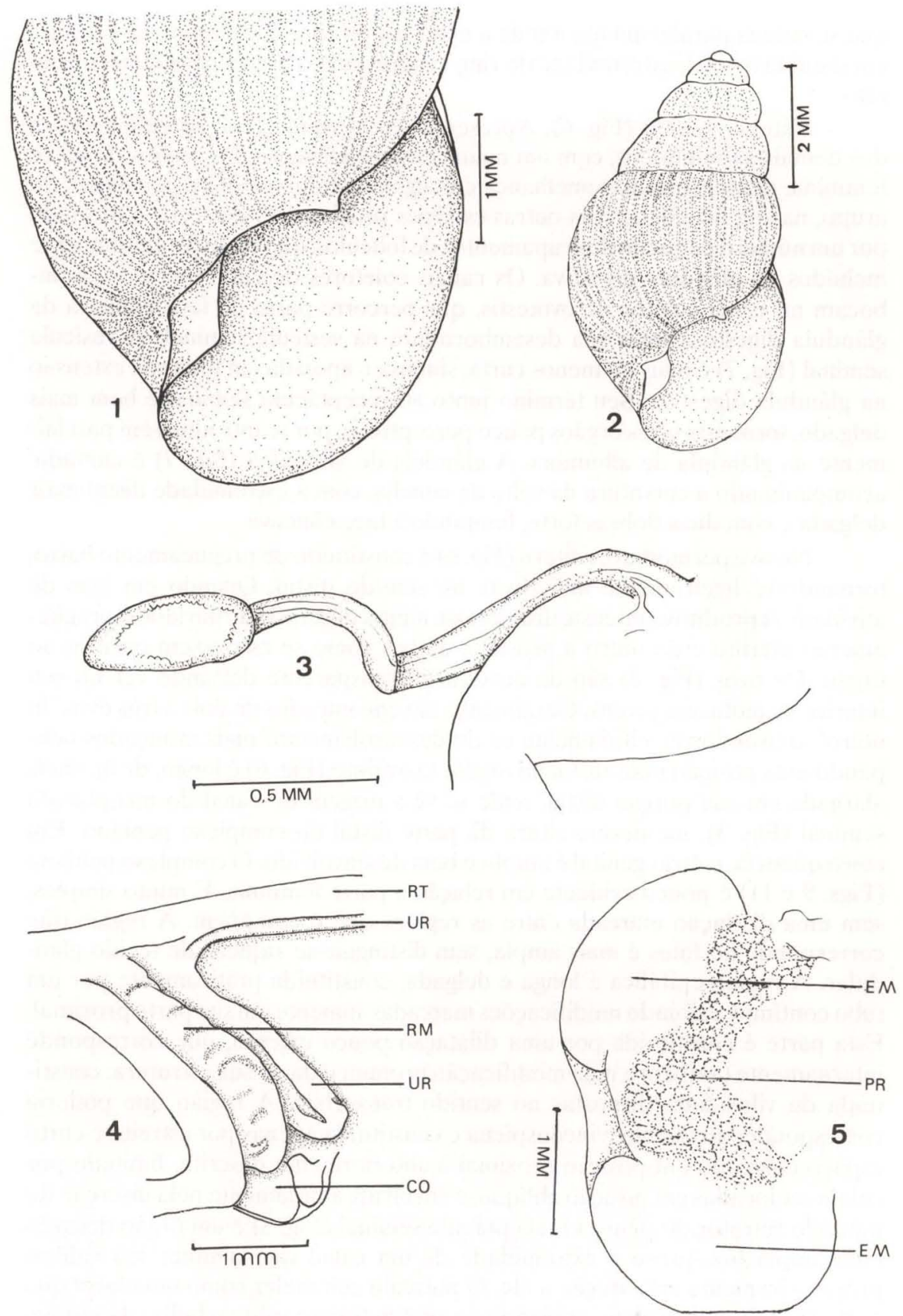

Figs 1-5. Leptinaria unilamellata. (1) Abertura da concha; (2) concha; (3) canal do receptáculo seminal e receptáculo seminal; (4) porção proximal da câmara palial; (5) porção média do ovispermoduto. 
que se coloca paralelamente a toda a extensão do rim. A curvatura se faz logo em seguida à outra extremidade do rim, caminhando o ureter paralelamente ao reto.

Sistema genital (Fig. 6). Apresenta seu aspecto geral, aproximando-se dos demais subulinideos, com um maior desenvolvimento dos órgãos da parte feminina, demonstrando semelhança em algumas características peculiares do grupo, na sua fisiologia, com outras espécies próximas. O ovotestis é formado por um número pequeno de grupamentos de folículos, que estão profundamente incluídos na glândula digestiva. Os canais coletores de cada folículo desembocam no canal coletor de ovotestis, que percorre parte da face côncava da glândula digestiva até a sua desembocadura na vesícula seminal. A vesícula seminal (Fig. 7) é mais ou menos curta, sinuosa e apoiada em toda sua extensão na glândula digestiva. Seu término junto ao receptáculo seminal é bem mais delgado, tornando estes órgãos pouco perceptíveis, por se introduzirem parcialmente na glândula de albumina. A glândula de albumina (Fig. 7) é curvada, acompanhando a curvatura da volta da concha, com a extremidade distal mais delgada e com duas dobras forte, limitando a face côncava.

No ovispermoduto, o útero (Fig. 6) é constituido de pregueamento baixo, tornando-se ligeiramente mais forte no sentido distal. Quando em fase de atividade reprodutiva intensa, distende-se muito, deixando de um lado o pregueamento uterino e do outro a próstata, ambos como se estivessem colados ao órgão. Os ovos (Fig. 8) são de envoltório transparente deixando ver no seu interior os moluscos jovens. Geralmente são encontrados de dois a três ovos "in utero", deixando ver nitidamente os de desenvolvimento mais avançados ocupando uma posição mais distal no órgão. O oviduto (Fig. 6) é longo, de luz mais alargada em sua porção distal, onde se vê a origem do canal do receptáculo seminal (Fig. 3), na mesma altura da parte distal do complexo peniano. Em consequiência, o átrio genital é amplo e bem desenvolvido. O complexo peniano (Figs. 9 e 11) é pouco evidente em relação à parte feminina. É muito simples, sem uma distinção marcada entre as regiões que o compõem. A região que corresponde ao falus é mais ampla, sem distinguir-se riqueza de tecido glandular. A região epifálica é longa e delgada, constituida praticamente por um tubo contínuo, exibindo modificações marcadas somente em sua parte proximal. Esta parte é constituida por uma dilatação pouco extensa, que corresponde internamente também a uma modificação pronunciada de sua estrutura, constituída de vilosidade dispostas no sentido transversal. A região que poderia corresponder ao flagelo é inconspícua e constituida apenas por estreito e curto espaço colocado em posição proximal à anteriormente descrita, limitado por células colocadas em posição oblíqua, e envolvida amplamente pela inserção do músculo retrator do pênis. O receptáculo seminal (Fig. 3) é um órgão delgado com ampla luz, preso à extremidade de um canal que diminui seu calibre progressivamente em direção a ele. $\mathrm{O}$ músculo columelar como uma faixa que se destaca. É muito longo, apoiando-se em seu trajeto sobre o bulbo da rádula, contornando-o parcialmente em sua parte superior, passando por baixo do tentáculo esquerdo e inserindo-se terminalmente no complexo peniano, envol- 


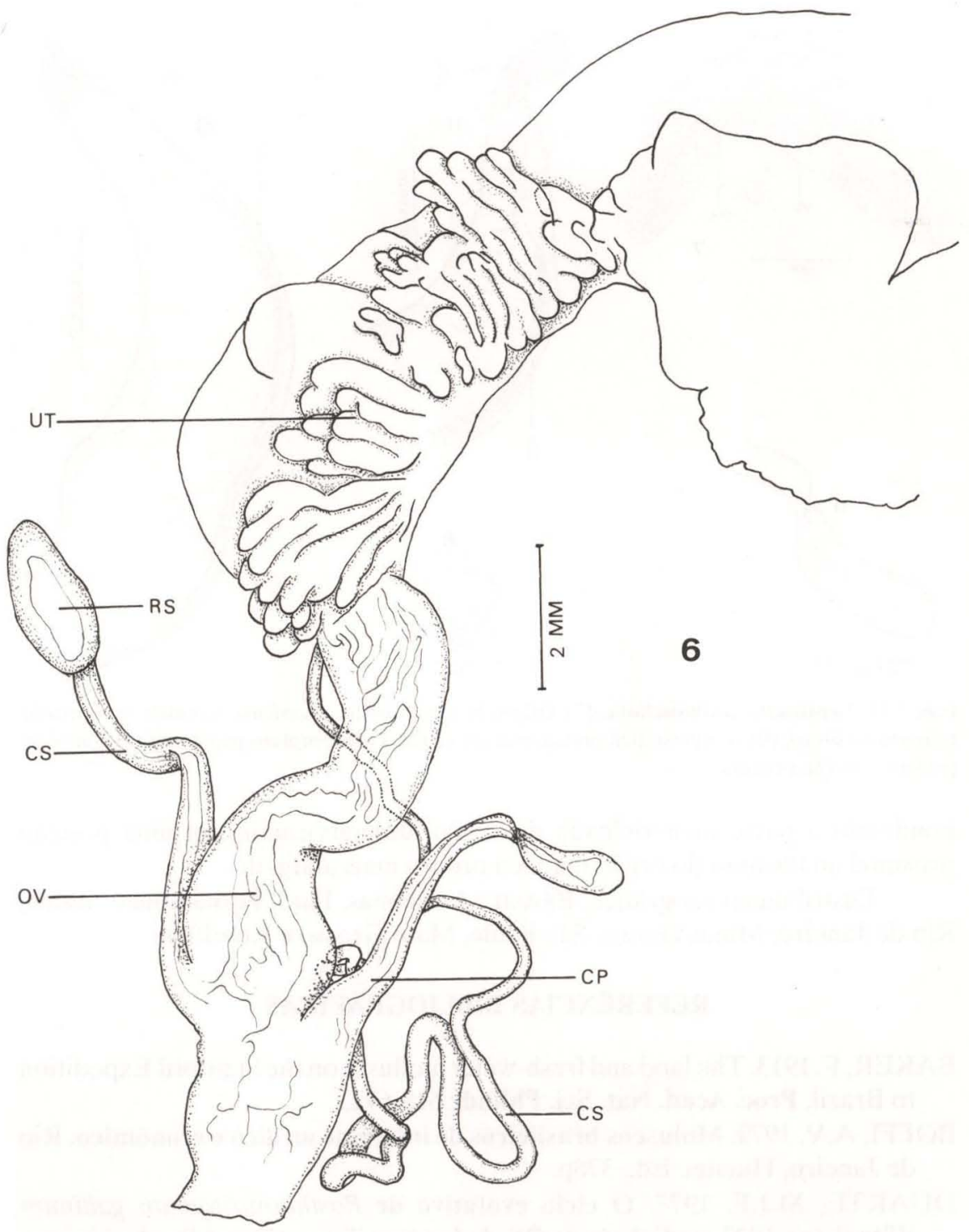

Fig. 6. Leptinaria unilamellata. Aparelho genital, porções média e distal.

vendo-o amplamente. $O$ canal deferente (Figs 6, 10,11) é a parte mais marcante do sistema masculino, iniciando-se na porção distal da próstata, onde também termina o pregueamento uterino, prende-se ao oviduto ao cruzá-lo e se dirige para a porção distal do falus, aumenta de calibre ao mesmo tempo que se torna muito enovelado, mantendo-se preso por tecido conjuntivo frouxo, junto ao complexo peniano, com sua terminação delgada, como no início. Em seguida caminha paralelamente à região epifálica por uma distância bem longa, corres- 

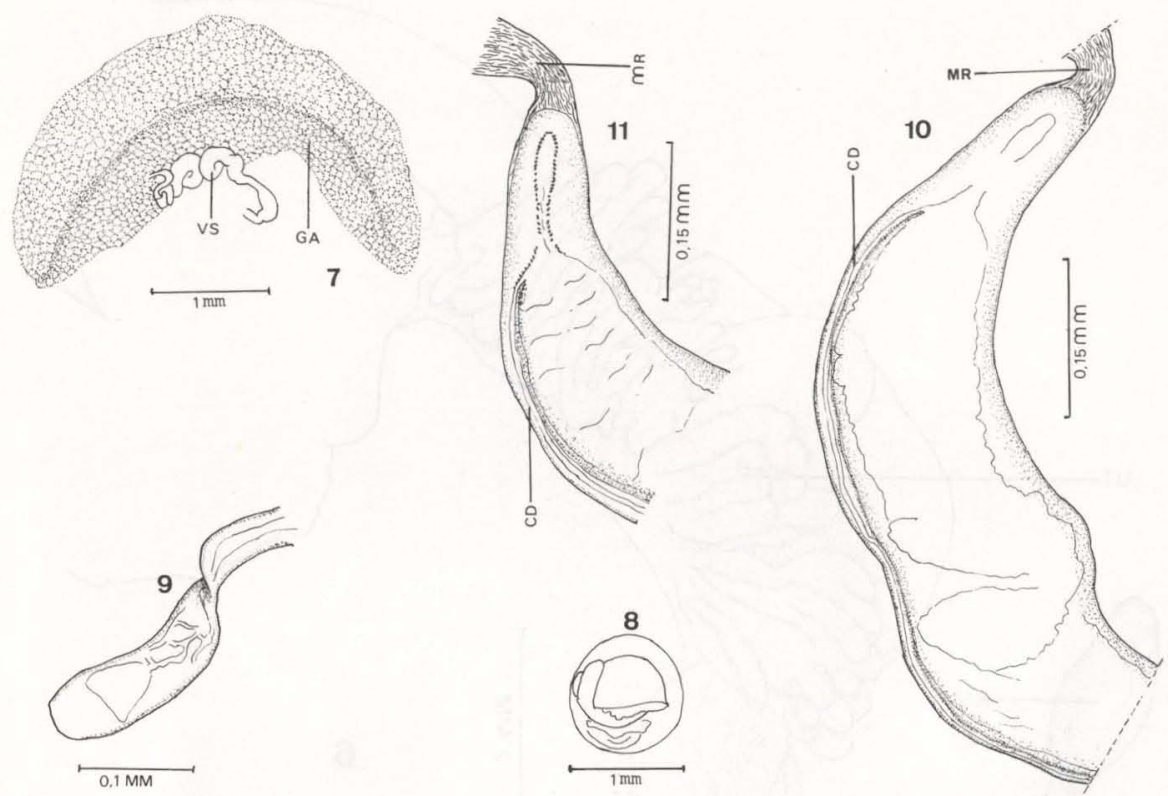

Figs 7-11. Leptinaria unilamellata. (7) Glândula de albumina e vesícula seminal; (8) embrião retirado do útero; (9) complexo peniano, porção proximal; (10) complexo peniano; (11) complexo peniano, porção proximal.

pondendo à parte mais delgada do órgão, desembocando em uma posição proximal ao término do órgão em uma proção mais alargada.

Distribuição geográfica. BRASIL: Amazonas, Pará, Pernambuco, Bahia, Rio de Janeiro, Minas Gerais, São Paulo, Mato Grosso e Rondônia.

\section{REFERÊNCIAS BIBLIOGRÁFICAS}

BAKER, F. 1913. The land and fresh-water mollusks on the Stanford Expedition to Brazil. Proc. Acad. Nat. Sci. Philad.: 618-672.

BOFFI, A.V. 1979. Moluscos brasileiros de interesse médico e econômico. Rio de Janeiro, Hucitec Ed., 376p.

DUARTE, M.J.F. 1977. O ciclo evolutivo de Postharmostomum gallinum Witenberg, 1923, no Estado do Rio de Janeiro. Tese, não publicada, Universidade Federal Rural do Rio de Janeiro, Itaguaí, 69p.

HAAS, F. 1952. South American non-marine shells: further remarks and descriptions. Field. Zool. 34 (9): 107-132.

. 1962. Caribbean Land Molluscs: Subulinidae and Oleacinidae, Studies on the fauna of Curaçao and other Caribbean Islands. XIII (58): 49-60. The Hague Martius Nijhoff.

KELLER, D.G. \& J.L. DE B. ARAUJO. (no prelo). Ciclo evolutivo de Paratanaisia bragai (Santos, 1934) (Trematoda, Eucotylidae), com um novo 
hospedeiro intermediário no Brasil: Leptinaria unilamellata (Orbigny, 1835) (Gastropoda, Pulmonaata, Subulinidae) em condições de laboratório.

MORRETES, F.L. 1943. Contribuição ao estudo da fauna brasileira de moluscos. Pap. Av. Depto. Zool. 3 (7): 111-126.

OLIVEIRA, M.P.; G.J.R. REZENDE \& G.A. CASTRO. 1981. Catálogo dos moluscos da Universidade Federal de Juiz de Fora. Juiz de Fora, Universidade Federal de Juiz de Fora Ed., 520p.

ORBIGNY, A.D'. 1835/1846. Voyage dans l'Amerique Meridionale. Paris. Mollusques 5 (3): 185-376 (1837); 489-758 (1846).

PFEIFFER, L. 1848. Monographia Heliceorum Viventium. 2: 1-594.

1859. Monographia Heliceorum Viventium. 4: 1-920.

PILSBRY, H.A. 1933. Zoological results of the Matto Grosso expedition to Brazil in 1931. II - Mollusca. Proc. Acad. Nat. Sci. Philad. 85: 67-76.

REEVE, L.A. 1849. Conchologia Iconica. Illustrations of the shells of molluscous animals. Vol. V.

TILLIER, S. 1989. Comparative morphology and classification of land snails and slugs (Gastropoda: Pulmonata: Stylommatophora). Malocologia 30 (1-2): 1-303.

THIELE, J. 1931. Handbuch der Systematischen Weichtierkunde. 1. Jena, $\mathrm{VI}+778 \mathrm{p}$.

TRYON, G.W. \& PILSBRY,H.A. 1906. Manual of Conchology. XVIII: I-VII + $357 \mathrm{p}$.

VON MARTENS, E. 1890/1901. Biologia Centrali-Americana. Land and Freshwater mollusca. XXVIII + 705p.

WENS, W. 1960. Handbuch der Systematischen Palaozoologie, Gastropoda, Euthyneura 6 (2): XII + 834, Berlin. 